\title{
Validity of the Brazilian version of the freezing of gait questionnaire
}

\author{
Validação da versão brasileira da escala de congelamento da marcha \\ Jussara A. Oliveira Baggio, Mônica B. Curtarelli, Guilherme R. Rodrigues, Vitor Tumas
}

\begin{abstract}
Objective: To validate the freezing of gait questionnaire (FOG-Q) for a Brazilian population of Parkinson's disease (PD) patients. Methods: One hundred and seven patients with a diagnosis of PD were evaluated by shortened UPDRS motor scale (sUPDRm), Hoehn and Yahr (HY), Schwab and England scale (SE), Berg balance scale (BBS), falls efficacy scale international (FES-I), gait and balance scale (GABS), and the FOG-Q Brazilian version. Results: $47.7 \%$ of PD patients had FOG episodes; this group had worse scores on sUPDRSm, FOGQ, FES-I, BBS, GABS and FOG item of UPDRS when compared to the PD group without FOG. The internal consistency was 0.86 , intra-rater 0.82 and inter-rater 0.78 . The FOG-Q Brazilian version was significantly correlated with items related to gait and balance. The ROC curve was 0.94 , the sensitivity was 0.90 and specificity was 0.92 . Conclusion: Our study suggests that the FOG-Q Brazilian version is a reliable and valid instrument for assessing FOG in PD patients.
\end{abstract}

Key words: gait, freezing, scale, validation studies, Parkinson's disease.

\section{RESUMO}

Objetivo: Validar a escala de congelamento da marcha (FOG-Q) para a população brasileira com doença de Parkinson (DP). Métodos: Cento e sete pacientes com diagnóstico de DP foram avaliados através da versão reduzida da escala UPDRS motora (UPDRSm), HY (Hoehn e Yahr), SE (Schwab e England), escala de equilíbrio de Berg (BBS), escala internacional de medo de quedas (FES-I), escala de marcha e equilíbrio (GABS) e FOG-Q. Resultados: 47,7\% dos pacientes com DP foram identificados com congelamento da marcha. Este grupo obteve resultados significativamente piores nas UPDRSm, FOGQ, FES-I, BBS, GABS e no item de congelamento da UPDRS em comparação ao grupo sem o sintoma. A consistência interna da escala foi 0,86, a confiabilidade inter-examinador 0,82 e intra-examinador 0,78. Curva ROC de 0,94, sensibilidade 0,90 e especificidade 0,92. Conclusão: Nosso estudo demonstra que a versão brasileira da escala de congelamento da marcha é válida para avaliar o congelamento da marcha em pacientes com DP.

Palavras-Chave: marcha, congelamento, estudos de validação, doença de Parkinson.

Freezing of gait (FOG) is a common and disabling symptom in patients with Parkinson's disease (PD). It is characterized by a sudden inability to initiate or to continue walking, and is described by patients as a situation in which their feet seem to be "stuck to the ground" despite attempts to walk" There are some situations where FOG is more commonly induced, such as at the initiation of gait, during turning while walking, as passing through narrow spaces, or immediately before reaching a destination ${ }^{2}$. However, the clinical presentation of FOG is very variable, unpredictable, and has complex relationship with the antiparkinsonian medications ${ }^{1,3}$. It also depends on the psychological state of the patient that may be distinctly influenced by the degree of stressful conditions and by the balance between attention and distraction during walking². FOG may be a challenge for clinicians, since in many times it may be difficult to identify and assess the problem. For the clinical diagnosis, it is recommended to direct the history taking specifically for the presence of freezing, and to evaluate gait during a trajectory that features specific triggers to elicit FOG in ON and OFF state ${ }^{3}$.

Recently, Giladi et al. ${ }^{4}$ developed a clinical questionnaire to assess FOG in parkinsonian patients. The freezing of gait questionnaire (FOG-Q) has 6 questions and the total score ranges from 0 to 24; higher scores correspond to more severe FOG. It is a useful tool for history taking of clinical data suggesting the presence of freezing. The item 3 of the scale directly assesses the presence of freezing. This scale proved to be reliable to screen and measure the severity of FOG in

Department of Neuroscience and Behavior, Faculty of Medicine at Ribeirão Preto, Universidade de São Paulo, Ribeirão Preto SP, Brazil.

Correspondence: Jussara A. Oliveira Baggio; Department of Neuroscience and Behavior, School of Medicine at Ribeirão Preto, Universidade de São Paulo; Avenida Bandeirantes 3900; 14049-900 Ribeirão Preto SP - Brasil; E-mail: jussaraoliv@usp.br

Support: Coordenação de Aperfeiçoamento de Pessoal de Nivel Superior (CAPES).

Conflict of interest: There is no conflict of interest to declare.

Received 15 November 2012; Received in final form 12 April 2012; Accepted 19 April 2012 
patients with $\mathrm{PD}$, and also to assess treatment interventions ${ }^{4}$. The main limitation of this approach is that it does not include the definite identification of freezing, hereby, the presence of false-positive and negative freezers cannot be totally excluded. The main objective of this study was to validate a Brazilian version of the FOG-Q.

\section{METHODS}

\section{Cross-cultural adaptation of the FOG-Q}

We obtained authorization from the original authors to validate the scale. The FOG-Q was independently translated by two health professionals that were native Portuguese speakers and well acquainted with the English language (J.A.O.B. and V.T.). The two translated versions were then compared and adapted to get a final consensual version. Then, this version was back-translated into English by a native English speaker that was familiar with the Portuguese language and who had not previous contact with the original version of the scale. The back-translated version was compared to the original version of the FOG-Q by the translators, who examined the differences and made consensual modifications.

\section{Patients}

We evaluated 107 patients with a diagnosis of PD according to the UK Brain Bank diagnostic criteria that consecutively attended the Movement Disorders Outpatient Clinic of the Ribeirão Preto School of Medicine.

We excluded patients with other associated motor disorders (e.g., stroke), dementia according to the DSM-IV diagnostic criteria, concomitant severe systemic illness, acute disorders or injuries, severe sensorial deficits (e.g., blindness) or evident peripheral neuropathy, and orthopedic conditions that could interfere with gait.

This study was approved by ethics committee of Universidade de São Paulo (Comitê de Ética em Pesquisa CEP - Protocol no 2.913/2008). Written informed consent was obtained from all of the subjects.

\section{Clinical assessments}

The patients were all evaluated during the ON state, firstly by a neurologist that used the Hoehn and Yahr staging $(\mathrm{HY})^{5}$ and the Schwab and England functional scale (SE) ${ }^{6}$. He also rated the patient's signs and symptoms using a shortened version of the unified Parkinson's disease rating scale (UPDRS), which included a motor score that measured the same motor signs as the short Parkinson's evaluation scale but with the original five-point score of the UPDRS ${ }^{7}$. This shortened version of the UPDRS motor scale (sUPDRSm) was shown to have good reliability and validity in Brazilian patients with $\mathrm{PD}^{7}$.

After that, one of the authors (J.A.O.B.) interviewed the patients and recorded demographic and clinical information and history of falls in the previous twelve months. A "fall" was defined as an event resulting in a person coming to rest unintentionally on the ground or on another level ${ }^{8}$.

Subsequently, the same examiner described for the patient a FOG episode as a situation in which their feet seem to be "stuck to the ground". In some cases, it was necessary to demonstrate a FOG episode to be sure that the patient understood what it is. After that, the examiner applied the FOG-Q and the patients completed the falls efficacy scale international (FES-I) ${ }^{9}$. This scale was previously validated for the Brazilian population and it is a self-administered tool that measures the concern about falling during the performance of a range of daily living activities ${ }^{10}$. Patients were also evaluated by the same examiner using the Brazilian version of the Berg balance scale (BBS) ${ }^{11}$ and the clinical gait and balance scale (GABS). The GABS include a specific item (item 22 ) to evaluate the presence of freezing during a provocative maneuver of rising from a chair, walking 5 meters between 2 chairs spaced $24 \mathrm{in}$. apart, turning $180^{\circ}$, and walking back and sitting down. The total score vary from 0 to 5 , and a score of 1 is already indicative for the presence of freezing.

Ten patients were evaluated by the same examiner one week apart for the determination of intra-rater reliability and they were also evaluated by other examiner for determination of inter-rater reliability.

The definite presence of FOG to classify the patient as a freezer was based on the final clinical impression of the examiners and it was grounded in the score of item 14 of UPDRS (score $>0$ ) or in the observation of FOG during testing the item 22 of GABS.

\section{Statistical analysis}

Descriptive statistics was used to analyze the clinical and demographic characteristics of all participants. For comparisons between groups, we used the Mann-Whitney test.

The internal reliability of the FOG-Q Brazilian version was assessed using Cronbach's alpha, and item analyses were conducted by examining the effect on Cronbach's alpha of excluding each of the six FOG-Q items individually. Values $>0.70$ were considered to be acceptable. The test-retest reliability was assessed using intraclass correlation coefficient (ICC) and stability was considered acceptable when values $\geq 0.70$ for inter- and intra-rater reliability were reached.

Convergent validity represents the extent to which a measure is related to other variables conceptually associated with the same construct, and was evaluated by means of Spearman rank correlation coefficient $\left(\mathrm{r}_{\mathrm{s}}\right)$. We calculated the correlation between the FOG-Q scores and the single UPDRS item specifically addressing FOG (Part II - ADL item 14), the freezing item of the GABS (item 22), the sum score of the axial items of the sUPDRSm (speech, rising from a chair, gait and postural stability), and with other clinical instruments and demographic data. 
We calculated the area under the receiver operating characteristics (ROC) curve for the total score of the FOG-Q.

We made comparisons between FOG-Q item 3, UPDRS Part II (ADL-FOG) item 14 and FOG item of the GABS (item 22) using Kappa static.

The level of statistical significance was set at $\mathrm{p}<0.05$.

\section{RESULTS}

The 107 patients with PD evaluated had a median age of 62 years (range 33-83), a median of 7 years of disease duration (range 3-28), a median HY stage of 2 (range 1-4) and a median SE score of 80 (range 30-100).

Based on the final clinical impression, 51 patients (47.7\%) with PD were classified as freezers and 56 patients (52.3\%) were diagnosed as non-freezers. The clinical and demographic characteristics of these patients are presented in Table 1.

Internal consistency of the FOG-Q as measured by Cronbach's alpha was 0.86 and item analysis indicated that all FOG-Q items contributed to these high reliability values, since the exclusion of any of the items from the scale would not reduce alpha to below this value.

Test-retest and inter-rater reliability of the FOG-Q total score were respectively 0.78 and 0.82 .

The correlation coefficients between the FOG-Q and the other clinical evaluations are summarized in Table 2. The
Table 2. Correlation coefficients for the freezing of gait questionnaire.

\begin{tabular}{|c|c|c|}
\hline & $\begin{array}{l}\text { Correlation } \\
\text { coefficients }\left(r_{s}\right)\end{array}$ & $p$-value \\
\hline Disease duration & 0.44 & $0.0001 *$ \\
\hline Age & -0.21 & $0.024^{*}$ \\
\hline $\begin{array}{l}\text { Number of falls in the last } 12 \\
\text { months }\end{array}$ & 0.21 & $0.027 *$ \\
\hline FES-I & 0.32 & $0.001 *$ \\
\hline SE score & -0.28 & $0.004^{*}$ \\
\hline HY stage & 0.22 & $0.02 *$ \\
\hline sUPDRSm (axial items) & 0.35 & $0.0001 *$ \\
\hline sUPDRSm & 0.16 & 0.1 \\
\hline GABS & 0.37 & $0.0001 *$ \\
\hline BBS & -0.27 & $0.005^{\star}$ \\
\hline UPDRS Part II (ADL-FOG) (item 14) & 0.79 & $0.0001 *$ \\
\hline FOG item of the GABS (item 22) & 0.36 & $0.0001 *$ \\
\hline \multicolumn{3}{|l|}{ GABS timed tasks } \\
\hline Walking 5 meters & 0.21 & $0.02 *$ \\
\hline Stand-walk-sit (10 meters) & 0.20 & $0.03 *$ \\
\hline Number of steps & 0.19 & $0.04^{*}$ \\
\hline $\begin{array}{l}\text { Walking as fast as possible } \\
\text { ( } 5 \text { meters) }\end{array}$ & 0.11 & 0.24 \\
\hline Cadence & -0.10 & 0.28 \\
\hline
\end{tabular}

HY: Hoehn and Yahr; SE: Schwab and England Scale; sUPDRSm: shortened UPDRS motor scale; FOG-Q: freezing of gait questionnaire; UPDRS: unified Parkinson's disease rating scale; GABS: gait and balance scale; FES-I: falls efficacy scale international; BBS: Berg balance scale; *FOG-Q score significantly correlated.

Table 1. Clinical and demographic characteristics of Parkinson's disease patients with and without FOG.

\begin{tabular}{|c|c|c|c|c|c|}
\hline & \multicolumn{4}{|c|}{ Patients with PD } & \multirow{3}{*}{$p$-value } \\
\hline & \multicolumn{2}{|c|}{$\begin{array}{l}\text { Non-freezers } \\
n=56(52.3 \%)\end{array}$} & \multicolumn{2}{|c|}{$\begin{array}{c}\text { Freezers } \\
\mathrm{n}=51(47.7 \%)\end{array}$} & \\
\hline & Mean (SD) & Median & Mean (SD) & Median & \\
\hline Age & $63.9(11.2)$ & 65 & $59.7(12.0)$ & 60 & 0.08 \\
\hline Disease duration & $7.2(5.2)$ & 7 & $9.0(4.2)$ & 7 & $0.002^{*}$ \\
\hline Hoehn and Yahr stage & $2.1(0.8)$ & 2 & $2.3(0.7)$ & 2.5 & 0.1 \\
\hline Schwab and England scale & $81.3(13.3)$ & 90 & $78.0(13.2)$ & 80 & 0.06 \\
\hline sUPDRSm & $11.4(6.2)$ & 10 & $13.8(6.7)$ & 12 & 0.05 \\
\hline$F O G-Q$ & $2.3(2.8)$ & 3 & $9.9(4.0)$ & 6 & $0.0001 *$ \\
\hline UPDRS Part II (ADL-FOG) (item 14) & $0.0(0.0)$ & 0 & $1.5(0.5)$ & 0 & $0.0001 *$ \\
\hline FOG item of the GABS (item 22) & $0.1(0.6)$ & 0 & $0.4(1.1)$ & 0 & $0.024^{\star}$ \\
\hline Number of falls in the last 12 months & $2.2(6.8)$ & 0 & $4.1(10.4)$ & 0 & 0.21 \\
\hline FES-I score & $23.4(11.3)$ & 18.5 & $29.8(14.0)$ & 21 & $0.01 *$ \\
\hline BBS score & $49.9(8.3)$ & 52 & $46.8(7.8)$ & 51 & $0.01 *$ \\
\hline GABS & $14.8(13.9)$ & 15 & $25.3(14.6)$ & 19 & $0.0001^{*}$ \\
\hline \multicolumn{6}{|l|}{ GABS timed tasks } \\
\hline Walking 5 meters & $7.0(2.6)$ & 6.4 & $9.2(5.3)$ & 7.1 & $0.01 *$ \\
\hline Number of steps & $11.3(3.2)$ & 11 & $14.1(6.2)$ & 12 & $0.006^{*}$ \\
\hline Cadence & $1.6(0.2)$ & 1.6 & $1.6(0.2)$ & 1.6 & 0.6 \\
\hline Walking as fast as possible ( 5 meters) & $5.5(2.4)$ & 5.2 & $6.6(3.9)$ & 5.5 & 0.1 \\
\hline Stand-walk-sit (10 meters) & $19.4(7.2)$ & 17.4 & $23.9(12.5)$ & 18.8 & $0.03^{*}$ \\
\hline
\end{tabular}

FOG: Freezing of gait; PD: Parkinson's disease; sUPDRSm: shortened UPDRS motor scale; FOG-Q: freezing of gait questionnaire; UPDRS: unified Parkinson's disease rating scale; GABS: gait and balance scale; FES-I: falls efficacy scale international; BBS: Berg balance scale; SD: standard deviation.

*Mann-Whitney test: significance level $<0.05$. 
FOG-Q score significantly correlated with duration of disease, age, number of falls over the previous 12 months, HY stage, FES-I, SE, BBS and GABS scores. It also correlated with most GABS timed tasks, except for cadence and time to walk five meters as fast as possible. The FOG-Q presented better correlated with axial items of the sUPDRSm than with the total sUPDRSm score.

The FOG-Q score had a significant and strong correlation with the freezing item of the UPDRS $\left(\mathrm{r}_{\mathrm{s}}=0.79\right.$; $\left.\mathrm{p}<0.0001\right)$ but a poor to moderate correlation with the freezing items of the GABS $\left(r_{s}=0.36 ; p<0.0001\right)$.

The area under the ROC curve for the FOG-Q was 0.94 (0.90-0.99). With a cut-off score of 4 points, the scale had sensitivity and specificity of 0.90 and 0.92 , respectively. Moreover, when the area under the ROC curve, sensitivity and specificity was calculated only for the item 3 of FOG-Q we found similar results. With a cut-off score of 1 point, the area under the ROC curve was 0.92 , and the item had a sensitivity of 0.92 and a specificity of 0.92 .

Item 3 of the FOG-Q (score $>0$ ) identified $47.6 \%$ of PD patients as freezers, while the item 14 of UPDRS Part II (ADL-FOG) identified $43.92 \%$ and the FOG item of the GABS (item 22) 13.08\%. This last item obtained a Kappa value of 0.158, demonstrating a slight agreement among the other two items, while between the item 14 of UPDRS Part II (ADL-FOG) and item 3 of the FOG-Q obtained Kappa of 0.88 , which indicates excellent agreement.

\section{DISCUSSION}

FOG is a frequent clinical problem in patients with $\mathrm{PD}^{12,13}$. Our study found that around $47 \%$ of patients with PD that attended a Brazilian specialized movement disorder clinic reported FOG. Other previous studies reported variable findings, and this large variability may be probably explained by methodological differences such as the criteria to identify FOG and by the clinical characteristics of the sample evaluated ${ }^{14-16}$.

Our patients with PD and FOG had a longer disease duration compared to patients without it, which is consonant with previous studies showing a strong association between FOG and the duration and severity of the disease ${ }^{4,17}$. They tended to be younger than those without FOG, which disagrees with previous findings showing that the frequency of FOG increases with age ${ }^{17,18}$. This may be possibly explained by the characteristics of our sample, recruited from a specialized clinic with younger and more severely affected patients.

As expected, our patients with FOG had increased fear of falling as measured by the FES-I. The fear of future falls restricts daily activities and mobility, which can lead to other complications, such as social isolation, depression and poor sleep quality ${ }^{19}$.
The Brazilian version of the FOG-Q that we proposed to validate had high internal consistency and test-retest reliability that were very similar to those described by other studies $^{4,20}$. This demonstrated that this version is a reliable and valid tool to assess FOG in PD.

The score of the FOG-Q correlated with measures of balance, gait and with the severity of the axial symptoms of PD. The convergent and divergent reliability of the Brazilian version of the FOG-Q was very similar to that observed in other studies, showing that patients with PD and FOG have more balance and gait problems and worse parkinsonian axial motor signs ${ }^{17,21}$. Because these symptoms directly interfere with mobility and social life, this may be one way by which FOG negatively affects quality of life ${ }^{22,23}$.

In contrast with previous studies, we found no significant correlations between the score of the Brazilian version of the FOG-Q and the total score of the sUPDRSm ${ }^{4,20}$. However, this could be explained by the summarized character of this brief scale.

The correlation found between the FOG-Q and item 22 of the GABS was poor, with the latter identifying only $13 \%$ of our population of PD patients as freezers. This result was much lower when compared to the FOG-Q and item 14 of the UPDRS Part II (ADL-FOG). Item 22 of the GABS is a direct and objective measure where FOG is scored after trying to evoke a freezing episode in different situations during the clinical evaluation, while item 14 of the UPDRS Part II (ADLFOG) and the FOG-Q are clinical questions that subjectively try to identify the phenomenon based on clinical information. We may conclude that in a clinical setting it is very difficult to reproduce a freezing episode due to its variability and unpredictability ${ }^{1}$.

When simple questions, item 3 of the FOG-Q and item 14 of UPDRS Part II (ADL-FOG), was compared to the FOG-Q total score, we observed similar results for the diagnosis of FOG. This suggests that the whole scale do not improve the screening of FOG. However, the FOG-Q is a very useful tool to quantify the severity of FOG episodes in patients with PD and to assess the efficacy of interventional studies, which justifies the use of the scale ${ }^{4,24}$.

Recently, new versions of the FOG-Q were made available. Nilsson et al. ${ }^{25}$ validated a self-administered version of the FOG-Q with good results compared to the original scale. Therefore, this version facilitates the investigation of FOG in mail-based surveys and clinical practice. Nonetheless, the reliability of this version in patients with different educational levels remains to be tested. Another version was developed by Nieuwboer et al. $^{26}$ and, in this case, the authors excluded the two items concerning gait difficulties and included questions about the impact of FOG on daily life.

The question of which is the best tool to identify FOG remains unanswered, and while we do not develop an automatic and continuous system to record locomotion 
and quantify FOG, the FOG-Q may successfully be used for that purpose. One of the limitations of the FOG-Q is that FOG is quantified according to patients' self-reports, and as shown in the study of Nieuwboer et al. ${ }^{26}$ patients tend to spontaneously underestimate the severity of FOG. However, the FOG-Q may also help to recognize and differentiate FOG from turning difficulties and offstate akinesia ${ }^{27}$.

In conclusion, the Brazilian version of the FOG-Q was shown to be a valid tool to assess FOG in patients with PD, as well as to have internal consistency and test-retest reliability similar to other versions of the scale.

\section{References}

1. Giladi N, Nieuwboer A. Understanding and treating freezing of gait in parkinsonism, proposed working definition, and setting the stage. Mov Disord 2008;23(Suppl 2):S423-S425.

2. Okuma Y, Yanagisawa N. The clinical spectrum of freezing of gait in Parkinson's disease. Mov Disord 2008;23(Suppl 2):S426-S430.

3. Snijders AH, Nijkrake MJ, Bakker M, Munneke M, Wind C, Bloem BR. Clinimetrics of freezing of gait. Mov Disord 2008;23(Suppl 2):S468-S474

4. Giladi N, Tal J, Azulay T, et al. Validation of the freezing of gait questionnaire in patients with Parkinson's disease. Mov Disord 2009;24:655-661.

5. Hoehn MM, Yahr MD. Parkinsonism: onset, progression and mortality. Neurology 1967;17:427-442.

6. Schwab R, England A. Projection technique for evaluating surgery in Parkinson's disease. Third Symposium in Parkinson's disease. Edinburgh, Livingstone, 1969:152-157.

7. Tumas V, Ujikawa LT, Ferreira GM. Utility and reliability of a simplified clinical scale for Parkinson's disease. Arq Neuropsiquiatr 2004;62(Suppl 2):S220-S221.

8. Ashburn A, Stack E, Pickering RM, Ward CD. A community-dwelling sample of people with Parkinson's disease: characteristics of fallers and non-fallers. Age Ageing 2001;30:47-52.

9. Yardley L, Beyer N, Hauer K, Kempen G, Piot-Ziegler C, Todd C. Development and initial validation of the Falls Efficacy ScaleInternational (FES-I). Age Ageing 2005;34:614-619.

10. Camargos F. Adaptação transcultural e avaliação das propriedades psicométricas da Falls Efficacy Scale - International: um instrumento para avaliar o medo de cair em idosos. [master's thesis]. Belo Horizonte: Universidade Federal de Minas Gerais; 2007.

11. Miyamoto ST, Lombardi Junior I, Berg KO, Ramos LR, Natour J. Brazilian version of the Berg balance scale. Braz J Med Biol Res 2004:37:1411-1421.

12. Achiron A, Ziv I, Goren M, et al. Primary progressive freezing gait. Mov Disord 1993;8:293-297.

13. Atchison PR, Thompson PD, Frackowiak RS, Marsden CD. The syndrome of gait ignition failure: a report of six cases. Mov Disord 1993:8:285-292.
14. Coelho M, Marti MJ, Tolosa E, et al. Late-stage Parkinson's disease: the Barcelona and Lisbon cohort. J Neurol 2010;257:1524-1532.

15. Giladi N, Treves TA, Simon ES, et al. Freezing of gait in patients with advanced Parkinson's disease. J Neural Transm 2001;108:53-61.

16. Lamberti P, Armenise S, Castaldo V, et al. Freezing gait in Parkinson's disease. Eur Neurol 1997;38:297-301.

17. Bloem BR, Hausdorff JM, Visser JE, Giladi N. Falls and freezing of gait in Parkinson's disease: a review of two interconnected, episodic phenomena. Mov Disord 2004;19:871-884.

18. Jankovic J, Kapadia AS. Functional decline in Parkinson disease. Arch Neurol 2001;58:1611-1615.

19. Adkin AL, Frank JS, Jog MS. Fear of falling and postural control in Parkinson's disease. Mov Disord 2003;18:496-502.

20. Nilsson MH, Hagell P. Freezing of Gait Questionnaire: validity and reliability of the Swedish version. Acta Neurol Scand 2009;120:331-334.

21. Giladi N, McDermott MP, Fahn S, et al. Freezing of gait in PD:prospective assessment in the DATATOP cohort. Neurology 2001;56:1712-1721.

22. Moore O, Peretz C, Giladi N. Freezing of gait affects quality of life of peoples with Parkinson's disease beyond its relationships with mobility and gait. Mov Disord 2007;22:2192-2195.

23. Marras C, McDermott MP, Rochon PA, et al. Predictors of deterioration in health-related quality of life in Parkinson's disease: results from the DATATOP trial. Mov Disord 2008;23:653-659.

24. Lo AC, Chang VC, Gianfrancesco MA, Friedman JH, Patterson TS, Benedicto DF. Reduction of freezing of gait in Parkinson's disease by repetitive robot-assisted treadmill training: a pilot study. J Neuroeng Rehabil 2010;7:51.

25. Nilsson MH, Hariz GM, Wictorin K, Miller M, Forsgren L, Hagel P. Development and testing of a self administered version of the Freezing of Gait Questionnaire. BMC Neurol 2010;10:85.

26. Nieuwboer A, Rochester L, Herman T, et al. Reliability of the new freezing of gait questionnaire: agreement between patients with Parkinson's disease and their carers. Gait Posture 2009;30:459-463.

27. Stack EL, Ashburn AM, Jupp KE. Strategies used by people with Parkinson's disease who report difficulty turning. Parkinsonism Relat Disord 2006;12:87-92. 